\title{
Therapie der ambulant erworbenen aspirationsassoziierten Lungenabszesse
}

Zusammenfassung: Die Therapie von ambulant erworbenen aspirationsassoziierten Lungenabszessen ruht auf zwei Säulen: 1) der adäquaten antimikrobiellen Therapie und 2) der effektiven Drainage. Die kalkulierte antimikrobielle Therapie sollte aufgrund des Erregerspektrums aus einem Aminopenicillin/ $\beta$ Laktamasehemmer oder Clindamycin bestehen. Das auf grampositive Erreger eingeschränkte Spektrum des Clindamycins kann ggf. durch ein Cephalosporin der 2. Generation erweitert werden. Grundsätzlich sollte die antimikrobielle Therapie bis zur vollständigen Rückbildung der Abszesshöhle sowie der begleitenden Infiltrate fortgesetzt werden. Die Drainage besteht häufig spontan. Indikationen für den Einsatz zusätzlicher Drainagetechniken (bronchoskopische oder perkutane Drainage) können in seltenen Fällen bei klinischem Nichtansprechen und fehlender Rückbildung der Abszesshöhle bestehen. Chirurgische Notfallinterventionen betreffen im Wesentlichen schwere Blutungen. Zusammen mit einer adäquaten Drainage ist durch eine antimikrobielle Therapie eine Erfolgsrate von 75-90\% zu erwarten.

Treatment of Community-Acquired Lung Abscess Associated with Aspiration: The management of community-acquired lung abscess associated with aspiration is based on adequate antimicrobial treatment and effective drainage. Empiric antimicrobial treatment should cover basic microbial patterns and consist of aminopenicillin/ $\beta$-lactamase-inhibitor or clindamycin. Since the spectrum of clindamycin is restricted to Grampositive microorganisms combination treatment of clindamycin with a second-generation cephalosporin may be warranted. It is crucial to continue antimicrobial treatment until complete resolution of the abscess cavity and the corresponding infiltrates. Drainage is frequently present spontaneously. Additional techniques to ensure effective drainage include bronchoscopic and percutaneous drainage. These techniques are only infrequently indicated in case of clinical failure and of delayed cavity closure. Indications for surgical emergency interventions are mainly limited to severe pulmonary hemorrhage. The clinical success rate of adequate antimicrobial treatment together with effective drainage reaches $75-90 \%$.

\author{
S. Ewig ${ }^{1}$, H. Schäfer ${ }^{2}$ \\ ${ }^{1}$ Medizinische Universitäts-Poliklinik Bonn, \\ 2 Winterberg Kliniken Saarbrücken
}

\section{Einleitung}

Die Systematik der Lungenabszesse wurde von uns kürzlich in klinisch handlungsrelevanter Form an anderer Stelle dargestellt [1]. Dabei erschien uns besonders bedeutsam, auf die durch Intensivtherapie, Immunsuppression und neue Erreger veränderte Epidemiologie der Lungenabszesse hinzuweisen und eine diese Faktoren berücksichtigende Klassifikation zu erstellen. Kurz zusammengefasst, sind folgende fünf Formen der Lungenabszesse zu unterscheiden: ambulant erworbene Abszesse mit und ohne Aspiration, sekundäre Abszesse, Lungenabszesse unter schwerer Immunsuppression und nosokomiale Lungenabszesse.

Ambulant erworbene aspirationsassoziierte Lungenabszesse sind heute aufgrund des hohen Lebensstandards sowie einer frühzeitigen Therapie von respiratorischen Infektionen mit effektiven antimikrobiellen Therapien seltener geworden [24]. Ebenso ist die Prognose von Lungenabszessen bei adäquater Therapie relativ günstig. Dennoch bleiben Lungenabszesse mit einer relevanten Letalität verbunden. In neueren Serien liegt diese zwischen 10 und 28\% [3-6]. Es besteht die Möglichkeit, dass zumindest ein Teil dieser Letalität durch vermeidbare Komplikationen bedingt ist $[3,4,6,8,9]$. Darüber hinaus wird durch eine zunehmend geringer werdende Erfahrung im Umgang mit dieser Erkrankung eine inadäquate Therapie begünstigt.

Wir möchten daher die aktuelle Therapie der ambulant erworbenen aspirationsassoziierten Lungenabszesse (im Folgenden einfach „Lungenabszesse“) darstellen. Besonderes Augenmerk gilt dabei der adäquaten antimikrobiellen Therapie und den unterschiedlichen Formen der Drainagetherapie sowie ihrer Indikation und Durchführung.

\section{Grundlagen}

Die Therapie von Lungenabszessen ruht auf zwei Säulen: 1) der antimikrobiellen Therapie und 2) der Drainage. Letztere besteht häufig spontan. Chirurgische Notfallinterventionen betreffen im Wesentlichen schwere Blutungen. Eine Indikation zur chirurgischen Sanierung von verbleibenden Resthöhlen besteht nur noch in Einzelfällen. Defektheilungen mit Bronchiektasiebildung werden kaum mehr gesehen. 


\section{Antimikrobielle Therapie}

\section{Erregerspektrum}

Die häufigsten Erreger aspirationsassoziierter Abszesse, gegen die die antimikrobielle Therapie gerichtet sein muss, sind die folgenden [10-18]:

- Viridans-Streptokokken (besonders Streptococcus milleri),

- Staphylococcus aureus,

zusammen mit

- anaeroben Peptostreptokokken,

- Bacteroides spp. (B. melaninogenicus/oralis, B. asacharolyticus, B. ureolyticus) und

- Fusobacterium nucleatum.

Meist findet sich eine Vielzahl von anaeroben Keimisolaten gleichzeitig, Isolate einzelner anaerober Erreger stellen meist Fusobacterium spp. dar.

Seltener angetroffen werden Streptococcus pneumoniae und gramnegative Enterobakterien (GNEB). Obwohl für Lungenabszesse nicht eigens belegt, dürfte das Risiko für letztere Erreger vor allem bei Patienten erhöht sein, die eine oropharyngeale Kolonisation durch entsprechende Keime aufweisen.

\section{Penicillin G}

Penicillin G (und alternativ Tetracyclin) ist über Jahrzehnte hinweg in der initialen kalkulierten antimikrobiellen Therapie Mittel der Wahl gewesen [9,19-24]. Heute werden diese Substanzen aus folgenden Gründen nicht mehr empfohlen:

1. Penicillin-Resistenz durch $\beta$-Laktamase-bildende Stämme von Bacteroides spp. und Fusobacterium spp. Die klinische Relevanz dieser Resistenz ist jedoch nicht eindeutig gesichert.

2. fehlende Wirksamkeit gegen eine Reihe von gramnegativen Aerobiern.

\section{Clindamycin}

Drei kontrollierte Studien zur Therapie von Lungenabszessen haben Penicillin versus Clindamycin untersucht. In der ersten
Studie aus dem Jahre 1975 wurde kein statistisch signifikanter Unterschied zwischen Clindamycin und Penicillin hinsichtlich Dauer der Entfieberung (Median 4 bzw. 7 Tage), des Verschlusses der Abszesshöhle (3,5 bzw. 4,5 Wochen) sowie der radiologischen Normalisierung gefunden ( 8 bzw. 10 Wochen) [25]. Es zeigt sich jedoch ein Trend zugunsten von Clindamycin.

Zwei Studien aus den Jahren 1983 bzw. 1990 haben eine Überlegenheit von Clindamycin über Penicillin hinsichtlich der Rate der Therapieversager, der Fieberdauer, der Dauer eitrigen Sputums sowie der Rezidivraten gefunden [26,27] (Tab.1).

\section{Metronidazol}

Metronidazol alleine hat trotz seiner guten Wirksamkeit gegen anaerobe Keime eine hohe Versagerrate. In einer randomisierten Studie zum Vergleich von Metronidazol mit Clindamycin zeigten sich Versagerraten von $58 \%$ versus $10 \%$ [28]. Diese Ergebnisse gründen am ehesten in der primären Resistenz von Viridans-Streptokokken gegenüber Metronidazol. Daher sollte Metronidazol allenfalls in Kombination mit Penicillin zum Einsatz kommen [28,29].

\section{Aminopenicillin plus $\beta$-Laktamasehemmer}

In einer neueren multizentrischen offenen Studie wurden 57 Patienten mit ambulant erworbenen Lungenabszessen mit Amoxicillin plus Clavulansäure in einer Dosierung von $4 \times 1 \mathrm{~g}$ intravenös über mindestens 7 Tage, gefolgt von $2 \mathrm{~g} /$ Tag oral behandelt. Die Population schloss 27 Patienten ein, die bereits ein Therapieversagen mit anderen antimikrobiellen Regimen aufwiesen. Ein Therapieerfolg konnte in 52 Fällen (91\%) erzielt werden [30].

\section{Ungeeignete Substanzen}

Nicht empfohlen werden können Makrolide aufgrund der primären Resistenz von Fusobacterium spp., sowie Aminoglykoside, Aztreonam und Cotrimoxazol aufgrund fehlender oder mangelhafter Aktivität gegen anaerobe Keime [31].

Tab. 1 Kontrollierte Studien zum Vergleich von Clindamycin und Penicillin G in der Therapie von Lungenabszessen oder nekrotisierender Pneumonien

\begin{tabular}{|c|c|c|c|c|}
\hline \multirow[t]{2}{*}{ Endpunkte } & \multicolumn{2}{|l|}{ Clindamycin } & \multicolumn{2}{|l|}{ Penicillin G } \\
\hline & $\begin{array}{l}\text { Levison u. Mitarb. [26] } \\
\mathrm{n}=19\end{array}$ & $\begin{array}{l}\text { Gudiol u. Mitarb. [27] } \\
n=19\end{array}$ & $\begin{array}{l}\text { Levison u. Mitarb. [26] } \\
\mathrm{n}=20\end{array}$ & $\begin{array}{l}\text { Gudiol u. Mitarb. [27] } \\
\mathrm{n}=18\end{array}$ \\
\hline $\begin{array}{l}\text { Rate der Therapieversager } \\
\text { (n) \% }\end{array}$ & 0 & $(1 / 19) 5$ & $(4 / 20) 20$ & $(8 / 18) 66$ \\
\hline mittlere Fieberdauer, Tage & 4,4 & 6,4 & 7,6 & 7,2 \\
\hline $\begin{array}{l}\text { mittlere Dauer des eitrigen } \\
\text { Sputums, Tage }\end{array}$ & 4,2 & 3,9 & 8,0 & 7,3 \\
\hline $\begin{array}{l}\text { Rezidivraten } \leq 10 \text { Tage nach } \\
\text { Therapieende, (n) \% }\end{array}$ & & - & & - \\
\hline Therapie über 3 Wochen & $(0 / 7) 0$ & & $(1 / 4) 25$ & \\
\hline Therapie über 6 Wochen & $(0 / 6) 0$ & & $(0 / 5) 0$ & \\
\hline
\end{tabular}

Dosierungen: Levison u. Mitarb.: Clindamycin: $3 \times 600 \mathrm{mg}$ i.v., anschließend $4 \times 300 \mathrm{mg}$ oral; Penicillin $6 \times 1$ Mio. E i. v., anschließend $4 \times 750 \mathrm{mg}$ Pen V oral. Guidol u. Mitarb.: Clindamycin: $4 \times 600 \mathrm{mg}$ i. v., anschließend $4 \times 300 \mathrm{mg}$ oral; Penicillin $6 \times 2$ Mio. E i.v., anschließend $4 \times 750 \mathrm{mg}$ Pen V oral 


\section{Aktuelle Therapieempfehlungen}

Aufgrund des Erregerspektrums empfehlen wir eine der folgenden Regimes:

1. Aminopenicillin/ $\beta$-Laktamasehemmer (Amoxicillin/Clavulansäure $3 \times 1,2 \mathrm{~g}$ bis $2,2 \mathrm{~g}$ bzw. Ampicillin/Sulbactam $3 \times 1,5-3 \mathrm{~g})$

2. Clindamycin $(3-4 \times 600 \mathrm{mg})$

Das auf grampositive Erreger eingeschränkte Spektrum des Clindamycins kann ggf. durch ein Cephalosporin der 2.Generation (z.B. Cefuroxim $3 \times 2 \mathrm{~g}$ ) erweitert werden. Dies gilt insbesonders für Patienten, die ein Risiko für eine gramnegative bakterielle oropharyngeale Kolonisation aufweisen (höheres Alter, wiederholte Hospitalisationen, Alkoholismus).

\section{Erwartbare Erfolgsrate und Kriterien des Therapieerfolgs}

Zusammen mit einer adäquaten Drainage ist durch eine antimikrobielle Therapie eine Erfolgsrate von $75-90 \%$ zu erwarten. Kriterien eines Therapieansprechens umfassen:

1. Klinische Kriterien, d.h. Entfieberung und Rückgang der Sepsiszeichen. Ein Ansprechen nach diesen Kriterien ist innerhalb von ca. drei bis sieben Tagen zu erwarten. Der exakte Zeitpunkt der Beurteilung des Therapieansprechens ist nicht eindeutig festzulegen, da auch Fälle mit verzögertem Ansprechen beobachtet werden. Dennoch favorisieren wir aufgrund der Gefahr einer Sepsis bzw. einer pulmonalen Blutung bei unbeherrschter Infektion einen Zeitrahmen vergleichbar der Pneumonie (48-72 h).

2. Rückgang der Abszesshöhlen-Größe: diese kann zwei bis zwölf Wochen in Anspruch nehmen [32,33].

3. Vollständige Rückbildung der Abszesshöhle sowie der begleitenden Infiltrate: Diese ist sehr variabel und kann bis zu drei bis sechs Monate in Anspruch nehmen. Folgende Rückbildungszeiten sind beschrieben: $13 \%$ nach 2 Wochen, 44\% nach 4 Wochen, 59\% nach 6 Wochen, $70 \%$ nach 3 Monaten [33]. In dieser Untersuchung wurde ein inverser Zusammenhang zwischen der Geschwindigkeit der Rückbildung und der Abszessgröße beobachtet.

Mögliche Komplikationen umfassen: Ausbreitung der Infektion durch Entleerung der Abszesshöhle, schwere Sepsis, schwere pulmonale Blutungen sowie bronchopleurale Fistelbildung mit Empyem (siehe Abb.1).

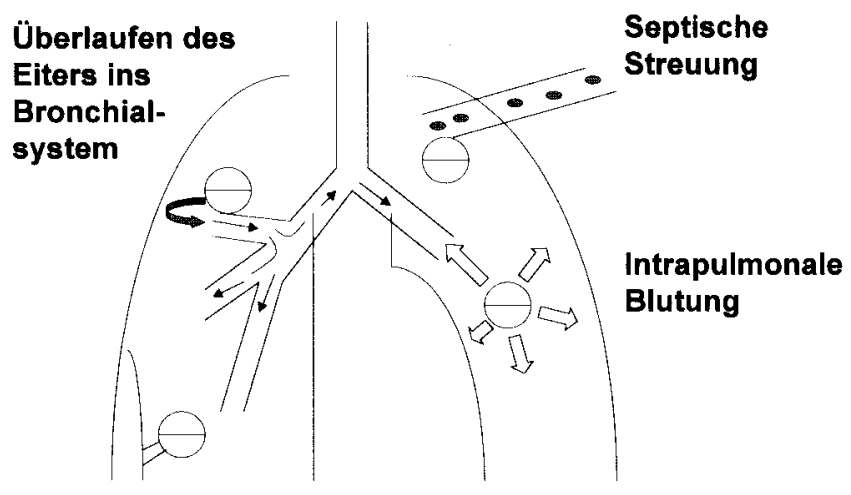

Bronchopleurale Fistel mit Empyem

Abb. 1 Mögliche Komplikationen des Lungenabszesses.
In einer neueren Arbeit, die 75 Patienten mit Lungenabszessen einschloss, wurden bei einer Letalität von $20 \%$ folgende Prädiktoren der Letalität identifiziert: Komorbidität, Anämie bei Aufnahme sowie Abszesse durch Staphylococcus aureus, Klebsiella pneumoniae und Pseudomonas aeruginosa [7].

\section{Dauer der antimikrobiellen Therapie}

Die Dauer der antimikrobiellen Therapie muss individuell gesteuert werden. Grundsätzlich sollte sie bis zur vollständigen Rückbildung der Abszesshöhle sowie der begleitenden Infiltrate fortgesetzt werden [32]. Eine vorzeitige Beendigung der Therapie kann zu Rezidiven führen. Dies bedeutet im Regelfall eine Therapiedauer von vier bis acht Wochen, in einzelnen Fällen aber auch von drei bis sechs Monaten. Initial sollte stets intravenös behandelt werden. Nach klinischem Ansprechen und radiologisch dokumentierter Rückbildung der Abszesshöhlen-Größe kann auf eine orale Therapie umgestellt werden.

\section{Drainagetherapie}

Der Drainage der Lungenabszesse kommt stets eine grundlegende Bedeutung zu nach dem Prinzip: „Ubi pus, ibi evacua“. In vielen Fällen besteht eine ausreichende spontane Drainage über einen Ableitungsbronchus, erkennbar am Gas-/ Flüssigkeitsspiegel und der großen Menge des expektorierten Eiters.

Das Bestehen einer effizienten Drainage sollte in der Regel bronchoskopisch gesichert werden. Bei leichtgradigen Verläufen und raschem Ansprechen auf die Therapie kann auf eine Bronchoskopie verzichtet werden. In gleicher Sitzung kann eine gründliche Bronchialtoilette durchgeführt werden. Es muss jedoch darauf hingewiesen werden, dass alle bronchoskopischen Eingriffe bei Patienten mit Lungenabszessen mit dem Risiko eines Übertritts von Eiter in bisher gesunde Lungenabschnitte auf der ipsi- und/oder kontralateralen Seite mit der möglichen Folge einer schweren Oxygenierungsstörung und Sepsis verbunden sind [34].

Hammer et al. haben daher folgende Vorsichtsmaßnahmen bei Bronchoskopien von Patienten mit Lungenabszessen empfohlen [34]:

1. Aufrechterhaltung der Wachheit sowie des Husten- und Schluckreflexes durch zurückhaltende Dosierung der Sedierung sowie der topischen Anästhesie,

2. geringstmögliche Manipulation der Abszesshöhle,

3. intensives Monitoring der Patienten post Bronchoskopie für mindestens $6 \mathrm{~h}$,

4. Lagerung des Patienten in Seitenlage (auf der erkrankten Seite)

Darüber hinaus ist die Durchführung einer bronchoalveolären Lavage bei Patienten mit Lungenabszessen kontraindiziert.

\section{Atemphysikalische Drainage}

Atemphysikalische Maßnahmen wie Lagerungsdrainage und Vibrationsmassage können zur effektiven Drainage beitragen. Auch das Erlernen von Techniken des effizienten Abhustens ist hilfreich. 


\section{Indikationen für zusätzliche Drainagetechniken}

Indikationen für den Einsatz zusätzlicher Drainagetechniken (bronchoskopische oder perkutane Drainage) sowie chirurgischer Maßnahmen (Lobektomie) können in seltenen Fällen bestehen bei [35]:

1. klinischem Nichtansprechen

2. fehlender Rückbildung der Abszesshöhle

Im Falle eines klinischen Therapieversagens nach einer adäquaten antimikrobiellen Therapie sowie erfolgter initialer Bronchoskopie sollte somit die Sicherstellung einer adäquaten Drainage erfolgen, sei es bronchoskopisch oder durch perkutane Drainage.

\section{Bronchoskopische Drainage}

Eine bronchoskopische Drainage ist möglich über:

1. (wiederholte) Bronchoskopien mit Absaugung unter Sicht Voraussetzung ist eine vorhandene freie Verbindung des Abszesses zu einem Ableitungsbronchus. Die Indikation zur (wiederholten) bronchoskopischen Absaugung ergibt sich bei mangelnder Fähigkeit, spontan abzuhusten.

2. Bronchoskopische Kathetereinlage

Die Indikation zu einer bronchoskopischen Kathetereinlage kann sich ergeben, wenn der Ableitungsbronchus durch eine ödematöse Schleimhautschwellung oder Fremdgewebe stenosiert ist. In diesem Fall ist die Einlage eines Katheters in den Ableitungsbronchus mit nachfolgender Absaugung möglich.

Erstmals von Metras u. Mitarb. 1954 beschrieben [36], liegen von 1975 bis 1988 vier Berichte zur bronchoskopischen Drainage mit $\geq 3$ Patienten vor. Insgesamt sind 27 Fälle beschrieben. In diesen Fällen bestand entweder kein suffizienter Ableitungsbronchus oder ein Therapieversagen. Entsprechend der experimentellen Natur dieser Technik wurden sehr unterschiedliche Katheter eingesetzt. Die Ableitungen waren überwiegend erfolgreich. Dokumentiert sind jedoch auch 5 Versager der Drainagetherapie bei Abzesshöhlen $<6 \mathrm{~cm}(36-40)$ (Tab. 2).
In einer Arbeit wurde über ein Verfahren berichtet, in dem ein Ableitungskatheter über einen bronchoskopisch platzierten Führungsdraht eingelegt, in situ belassen und anschließend dreimal täglich mit $25-50 \mathrm{ml} \mathrm{NaCl}$ gespült wurde. In allen drei Fällen kam es zur Entfieberung binnen 36 bis $48 \mathrm{~h}$. Allerdings kam es in einem dieser Fälle zur ungeplanten Katheterentfernung [40].

Insgesamt ist die Erfahrung mit der bronchoskopischen Drainage sehr begrenzt.

\section{Perkutane Drainage}

Eine perkutane Drainage kann chirurgisch oder unter Lokalanästhesie angelegt werden.

Indikationen für die perkutane Drainage können umfassen:

1. Nichtansprechen auf die antimikrobielle Therapie

- persistierende (7 bis 12 Tage nach Beginn der antimikrobiellen Therapie) oder neu aufgetretene Sepsis

- ausbleibende Entleerung der Abszesshöhle

2. Abszess-Komplikationen

- Spannungszeichen (fehlende Eiterspiegel, Gefahr der Eiteraspiration)

- bronchopleurale Fistel mit Empyembildung

Mögliche Komplikationen der perkutanen Drainage umfassen:

1. Pneumothorax

2. bronchopleurale Fistel mit Empyembildung

3. pulmonale Blutung

\section{Ergebnisse publizierter Berichte}

Insgesamt wurden von 1978 bis 1993 sieben Berichte zur perkutanen Drainage mit $\geq 3$ Patienten publiziert. Diese umfassen insgesamt 55 Patienten. Die Indikation zur Drainage bestand meist im Versagen der konservativen Therapie. Die Katheter-Einlageverfahren waren sehr heterogen und umfassten die Einlage nach Befund im Röntgen-Thorax oder in der CT des Thorax sowie die Einlage unter Durchleuchtungs- oder

Tab. 2 Ergebnisse der bronchoskopischen Drainagetherapie in Studien 1975- 1988 mit $\geq 3$ Patienten

\begin{tabular}{|c|c|c|c|c|c|}
\hline Autor & $\mathrm{n}$ & $\begin{array}{l}\text { Bronchoskopie-Indikation/ } \\
\text { Technik }\end{array}$ & Katheter & Erfolg & Komplikationen \\
\hline $\begin{array}{l}\text { Connors u. Mitarb., } \\
1975 \text { [37] }\end{array}$ & 3 & $\begin{array}{l}2 \times \text { Drainagebronchus nicht } \\
\text { unmittelbar zugänglich, } \\
1 \times \text { Therapieversagen/Starr }\end{array}$ & $\begin{array}{l}\text { Koronarkatheter rechte KA } \\
(8 \mathrm{~F}) \text { mit Teflon Führungsdraht }\end{array}$ & $3 / 3$ & keine \\
\hline $\begin{array}{l}\text { Rowe u. Mitarb., } \\
1979 \text { [38] }\end{array}$ & 10 & $\begin{array}{l}\text { Drainagebronchus nicht un- } \\
\text { mittelbar zugänglich/Flexibel }\end{array}$ & $\begin{array}{l}\text { Angiographiekatheter } \\
(0,047 \text { in.) }\end{array}$ & $\begin{array}{l}8 / 10 \text { sofort, } 7 / 10 \text { nach } \\
3 \text { Monaten }\end{array}$ & keine \\
\hline $\begin{array}{l}\text { Jeong u. Mitarb., } \\
1989 \text { [39] }\end{array}$ & 11 & $\begin{array}{l}\text { Therapieversagen nach } 1 \\
\text { Woche (physikalische und } \\
\text { am Therapie)/Flexibel }\end{array}$ & $\begin{array}{l}\text { Polyethylenkatheter } \\
\text { (Olympus PW-6P) }\end{array}$ & $\begin{array}{l}\text { 6/11 Entfieberung nach } \\
48 \mathrm{~h} \text { (alle mit Abszesshöhlen } \\
>8 \mathrm{~cm} / 2 \text { alternative Diagnosen) }\end{array}$ & $\begin{array}{l}5 \text { minimale } \\
\text { Hämoptosen }\end{array}$ \\
\hline $\begin{array}{l}\text { Schmitt u. Mitarb., } \\
1988[40]\end{array}$ & 3 & $\begin{array}{l}\text { Therapieversagen nach } \geq 3 \\
\text { Tagen am Therapie/Flexibel, } \\
\text { Dauerkatheter }\end{array}$ & $\begin{array}{l}\text { Führungsdraht - } 10 \mathrm{~F} \\
\text { Nephrostomie-Pigtail-Katheter } \\
(55 \mathrm{~cm} \text { lang); Spülung mit } \\
25-50 \mathrm{ml} \mathrm{NaCl} 3 \times / \text { Tag bis } \\
\text { klinische Besserung und } \\
\text { Abszesshöhle }<4 \mathrm{~cm}\end{array}$ & $\begin{array}{l}3 / 3 \\
\text { Entf. } 36 \mathrm{~h} / \text { Kath. } 1 \text { Woche } \\
\text { Entf. } 48 \mathrm{~h} / \text { Kath. ungeplant } \\
\text { entfernt nach } 48 \mathrm{~h} \\
\text { Entf. } 48 \mathrm{~h} / \text { Kath. } 4 \text { Tage }\end{array}$ & keine \\
\hline
\end{tabular}


Tab. 3 Ergebnisse der perkutanen Drainagetherapie in Studien 1978 - 1993 mit $\geq 3$ Patienten

\begin{tabular}{|c|c|c|c|c|c|c|}
\hline Autoren & $\mathrm{n}$ & Indikation & Verfahren & $\begin{array}{l}\text { Dauer bis } \\
\text { Entfieberung }\end{array}$ & Erfolg & Komplikationen \\
\hline $\begin{array}{l}\text { Vainrub u. Mit- } \\
\text { arb., } 1978[41]\end{array}$ & 3 & $\begin{array}{l}\text { Versagen der konservativen } \\
\text { Therapie (5-35 Tage), nicht } \\
\text { OP-fähig }\end{array}$ & perkutan, Durchleuchtung & k. A. & $100 \%$ & keine \\
\hline $\begin{array}{l}\text { Yellin u. Mitarb., } \\
1985 \text { [42] }\end{array}$ & 7 & $\begin{array}{l}\text { Versagen der konservativen } \\
\text { Therapie ( } 22-40 \text { Tage) }\end{array}$ & nach Rö-Thorax-Befund & $24-72 h$ & $100 \%$ & keine \\
\hline $\begin{array}{l}\text { Rice u. Mitarb., } \\
1987 \text { [43] }\end{array}$ & $\begin{array}{l}14(9 \\
\text { unter MV) }\end{array}$ & $\begin{array}{l}\text { Versagen der konservativen } \\
\text { Therapie [11], Spannungs- } \\
\text { zeichen [7] Weaning-Versagen } \\
\text { [4], Kontamination der kontra- } \\
\text { lateralen Lunge [4] }\end{array}$ & $\begin{array}{l}11 \text { perkutan (davon } 6 \text { nach } \\
\text { Rö-Thorax und } 3 \text { nach CT-Be- } \\
\text { fund, } 2 \text { mit Durchleuchtung), } \\
3 \text { chirurgisch }\end{array}$ & k. A. & $\begin{array}{l}11 / 14 \text { ( } 3 \text { ver- } \\
\text { storben, } 1 \text { abs- } \\
\text { zessassoziiert) }\end{array}$ & $\begin{array}{l}2 \text { minimale } \\
\text { Hämorrhagien }\end{array}$ \\
\hline $\begin{array}{l}\text { Parker u. Mitarb., } \\
1987 \text { [44] }\end{array}$ & 6 & $\begin{array}{l}\text { Versagen der konservativen } \\
\text { Therapie (11-33 Tage) }\end{array}$ & $\begin{array}{l}\text { perkutan, Durchleuchtung } \\
\text { (8-10 Fr Katether) }\end{array}$ & $48-72 h$ & $\begin{array}{l}4 / 6,1 \text { Besserung } \\
\text { nach Katheter- } \\
\text { revision, } 1 \text { Ver- } \\
\text { sager }\end{array}$ & keine \\
\hline $\begin{array}{l}\text { Shim u. Mitarb., } \\
1990 \text { [45] }\end{array}$ & 4 & $\begin{array}{l}\text { Versagen der konservativen } \\
\text { Therapie (9-25 Tage), nicht } \\
\text { OP-fähig }\end{array}$ & $\begin{array}{l}2 \text { Allgemeinanästhesie, } \\
2 \text { perkutan, Durchleuchtung }\end{array}$ & $24-48 h$ & $100 \%$ & keine \\
\hline $\begin{array}{l}\text { Van Sonnenberg } \\
\text { u. Mitarb., } 1991 \\
\text { [46] }\end{array}$ & 19 & $\begin{array}{l}\text { Versagen der konservativen } \\
\text { Therapie (bis } 22 \text { Tage) }\end{array}$ & perkutan, CT-gesteuert & bis $48 \mathrm{~h}$ & $100 \%$ & $\begin{array}{l}\text { Hämatothorax } \\
(n=1)\end{array}$ \\
\hline $\begin{array}{l}\text { Ha u. Mitarb., } \\
1993 \text { [47] }\end{array}$ & 5 & k. A. & perkutan, CT-gesteuert & $\begin{array}{l}\text { In } 5 / 6 \\
\text { binnen } 48 \mathrm{~h}\end{array}$ & $\begin{array}{l}80 \% \text { (1 Pat. mit } \\
\text { persist. Aspira- } \\
\text { tion) }\end{array}$ & keine \\
\hline
\end{tabular}

$\mathrm{MV}=$ maschinelle Beatmung, $\mathrm{k} . \mathrm{A}$. = keine Angaben

CT-Kontrolle. Die Erfolgsraten waren sehr hoch, abszessassoziierte Therapieversager wurden nur in drei Fällen dokumentiert (6\%) (Tab.3). Wichtig erscheint auch die Feststellung, dass kein einziger Fall eines Pleuraempyems nach perkutaner Drainageeinlage publiziert ist. Allerdings haben nicht alle Autoren in der Folge gleich gute Ergebnisse erzielen können (50\% Versagerrate bei Hirshberg u. Mitarb. [7]).

In der bisher größten Serie von 19 Patienten betrug die Erfolgsrate in Bezug auf die Drainage und Beherrschung der Sepsis 100\%. Nur 3/19 (16\%) der Patienten mussten anschließend aufgrund von Pleuraverwachsungen operativ saniert werden. Bei 18 von 19 Patienten kam es bereits innerhalb von $48 \mathrm{~h}$ zu einem Rückgang der Sepsiszeichen. In dieser Studie trat nur in einem Fall ein Hämatothorax als schwere Komplikation auf. In diesem Fall musste die Drainage durch gesunde Lunge geführt werden, darüber hinaus bestand eine leichte Blutungsneigung [46]. Nach einer perkutanen Drainage kann sich auch die Abszessgröße rasch vermindern, die vollständige Rückbildung der Abszesse nimmt jedoch mindestens $10-$ 14 Tage in Anspruch.

\section{Empfehlungen zur Technik der Drainageeinlage [35, 46]}

Voraussetzung für die perkutane Drainage ist der Nachweis eines pleuraständigen Abszesses. Das Passieren der gesunden Lunge im Rahmen der Kathetereinlage sollte aufgrund des Risikos der Hämorrhagie und der bronchopleuralen Fistel vermieden werden.

Technisch kann bei großen, der Pleura breitbasig aufliegenden Abszessen die direkte Platzierung des Katheters unter CT-
Kontrolle durchgeführt werden, bei kleineren Abszessen sollte die Seldinger-Technik bevorzugt werden. Bei der Auswahl des Katheters ist auf ein großes Lumen ( $\geq 12$ French) zu achten, da kleinlumige Katheter rasch verschließen. Von vitaler Bedeutung ist die korrekte Lagerung des Patienten: eine abhängige Lagerung der gesunden Seite ist aufgrund des Risikos eines Übertritts von Eiter in diese unbedingt $\mathrm{zu}$ vermeiden. Nach korrekter Platzierung sollte Aspirat asserviert und anschließend mit steriler Kochsalzlösung gespült werden. Der Katheter sollte anschließend an eine Drainage mit einem Sog von bis $\mathrm{zu}-20 \mathrm{~cm} \mathrm{H}_{2} \mathrm{O}$ angeschlossen werden. Um einen Verschluss des Katheters zu vermeiden, sollte mindestens zweimal pro Tag gespült werden. Der Katheter wird bis zur andauernden klinischen und/oder radiologischen Besserung in der Abszesshöhle belassen.

Indikationen für eine chirurgische Drainage mit Rippenresektion, wie sie von Monaldi beschrieben wurde, bestehen heute praktisch nicht mehr [48-51].

\section{Chirurgische Therapie}

Die chirurgische Therapie hat zwei Hauptindikationen:

1. die chirurgische Sanierung von Komplikationen

2. die chirurgische Resektion großer, unter konservativer Therapie nicht schließender Resthöhlen 


\section{Chirurgische Therapie der Komplikationen}

Die zwei chirurgisch angehbaren Komplikationen umfassen:

a) die intrabronchiale Blutung

b) das Empyem nach bronchopleuraler Fistelbildung. Das CT ist hier in der Unterscheidung wegweisend [52].

Schwere Blutungen (definierbar über Blutungsmenge pro Zeit) sind selten, jedoch stets lebensbedrohlich. Pathophysiologisch entstehen Blutungen innerhalb von Abszessen aufgrund von Nekrotisierungen von frischem gefäßreichen Granulationsgewebe. Thrombotisches Material verhindert oft eine suffiziente Spontandrainage, so dass der Entzündungsherd keine Entlastung erfährt. Auf diesem Hintergrund wird deutlich, dass hier ein Circulus vitiosus entstehen kann, der einer schweren Blutung den Weg bereitet.

Thoms u. Mitarb. haben drei im Röntgen-Thoraxbild erkennbare Zeichen einer bevorstehenden schweren pulmonalen Blutung herausgearbeitet $[53,54]$ :

- das Wiederauffüllen einer bereits entleerten oder leerer gewordenen Abszesshöhle („emptying-refilling“)

- die Umwandlung eines Luft-/Flüssigkeitsspiegels in eine (bewegliche) Masse („air fluid level to movable mass“)

- die persistierende Verdichtung mit fleckförmiger Aufhellungen („persistent radiodensity“)

Jedes dieser drei Zeichen repräsentiert eine Einblutung in die Abszesshöhle.

Ob allerdings im Falle des Vorliegens dieser Zeichen wie von den Autoren vorgeschlagen eine prophylaktische chirurgische Abszessentfernung gerechtfertigt ist, erscheint aufgrund der inzwischen verfügbaren Therapieoptionen fraglich. In jedem Fall rechtfertigen diese Befunde aber eine intensivere Überwachung des Patienten.

Im Falle einer schweren Blutung sollte bei Patienten mit ausreichender kardiopulmonaler Reserve primär eine Resektion (Lobektomie) angestrebt werden. Konservative Verfahren der Blutstillung können bei hinfälligen Patienten versucht werden.

Die Therapie des Empyems besteht primär in der Anlage einer Thoraxdrainage bzw. Saug-Spüldrainage. Im Falle des sonographischen Nachweises eines gekammerten Ergusses bzw. Empyems (Erguss- bzw. Empyemklasse 5 und 7 nach Light) kann die lokale Instillation von Fibrinolytika (z.B. Urokinase 100000 IE) versucht werden. Auf diese Weise lässt sich die Frühdekortikation meist vermeiden. Alternativ kann eine video-thorakoskopische Versorgung erfolgen. Eine Indikation zur chirurgischen Sanierung besteht bei konservativ nicht beherrschbarer Sepsis auf dem Boden der bronchopleuralen Fistelbildung.

\section{Chirurgische Resektion von Resthöhlen}

Auch die Entfernung von Resthöhlen wird heute nur noch ausnahmsweise erforderlich werden. Wichtig ist vor allem eine ausreichend lange Therapiedauer, da der Verschluss von größeren Abszesshöhlen $>3 \mathrm{~cm}$ auch längere Zeit als die erwarteten 3 Monate in Anspruch nehmen kann [32]. Aller- dings sollte im seltenen Falle eines Verbleibens einer Resthöhle trotz ausreichend langer Therapiedauer aufgrund des Risikos der sekundären Besiedlung durch Aspergillus spp. eine chirurgische Entfernung erwogen werden.

\section{Fazit}

Die Therapie der Lungenabszesse ist komplex und gehört in die Hände erfahrener Spezialisten. Adäquate kalkulierte initiale antimikrobielle Therapien umfassen ein Aminopenicillin/ $\beta$-Laktamasehemmer oder Clindamycin ( \pm Cephalosporin II) intravenös. Im Falle eines klinischen Therapieversagens nach einer adäquaten antimikrobiellen Therapie sowie erfolgter initialer Bronchoskopie sollte die Sicherstellung einer adäquaten Drainage erfolgen, sei es bronchoskopisch oder durch perkutane Drainage. Die Rolle der chirurgischen Therapie beschränkt sich weitgehend auf schwere Komplikationen wie z.B. schwere pulmonale Blutungen. Wichtig ist eine Fortsetzung der antimikrobiellen Therapie bis zur vollständigen Rückbildung der pulmonalen Affektionen.

\section{Literatur}

${ }^{1}$ Ewig S, Schäfer H. Lungenabszesse neu betrachtet. Eine klinisch handlungsanweisende Klassifikation. Pneumologie 2001; 55: $195-201$

${ }^{2}$ Schweppe HI, Knowles JH, Kane L. Lung abscess: an analysis of the Massachusetts General Hospital Cases from 1943 through 1956. N Engl J Med 1961; 265: 1039-1043

${ }^{3}$ Delarue NC, Pearson FG, Nellems JM, Cooper JD. Lung abscess: surgical implications. Can J Surg 1980; 23: 297-302

${ }^{4}$ Hagan JL, Hardy JD. Lung abscess revisited. A survey of 184 cases. Ann Surg 1983; 197: $755-762$

${ }^{5}$ Estrera AS, Platt MR, Mills LJ, Shwa RR. Primary lung abscess. J Thorac Cardiovasc Surg 1980; 79: 275-282

${ }^{6}$ Pohlson EC, McNamara JJ, Char C, Kurata L. Lung abscess: a changing pattern of disease. Am J Surg 1985; 150: 97 - 101

${ }^{7}$ Hirshberg B, Sklair-Levi M, Nir-Paz R, Ben-Sira L, Krivoruk V, Kramer MR. Factors predicting mortality of patients with lung abscess. Chest 1999; 115: 746-750

${ }^{8}$ Neild JE, Eykyn SJ, Philipps I. Lung abscess and empyema. Q J Med 1985; 224: 875-882

${ }^{9}$ Barnett TB, Herring CL. Lung abscess: initial and late results of medical therapy. Arch Intern Med 1971; 127: 217-227

${ }^{10}$ Barlett JG, Gorbach SL, Tally FP, Finegold SM. Bacteriology and treatment of primary lung abscess. Am Rev Respir Dis 1974; 109: 510-517

${ }^{11}$ Irwin RS, Garrity FL, Erickson AD, Corrao WM, Kaemmerlen JT. Sampling lower respiratory tract secretions in primary lung abscess. A comparison of the accuracy of four methods. Chest 1981; 79: 559-565

${ }^{12}$ Grinan NP, Lucena FM, Romero JV, Michavila IA, Dominguez SU, Alia CF. Yield of percutaneous needle lung aspiration in lung abscess. Chest 1990; 97: 69-74

${ }^{13}$ Henriquez AH, Mendoza J, Gonzalez P. Quantitative culture of bronchoalevolar lavage from patients with anaerobic lung abscesses. J Infect Dis 1991; 164: $414-417$

${ }^{14}$ Yang PC, Luh KT, Lee YT et al. Lung abscess: ultrasound examination and US-guided transthoracic aspiration. Radiology 1991; 180: $171-175$

${ }^{15}$ Wong CA, Donald F, Macfarlane JT. Streptococcus milleri pulmonary disease: a review and clinical description of 25 patients. Thorax 1995; 50: 1093-1096

${ }^{16}$ Hammond JMJ, Potgieter PD, Hanslo D, Scott H, Roditi D. The etiology and antimicrobial susceptibility patterns of microorga- 
nisms in acute community-acquired lung abscess. Chest 1995; 108: $937-941$

${ }^{17}$ Jerng JS, Hsueh PR, Teng LJ, Lee LN, Yang PC, Luh KT. Empyema thoracis and lung abscess caused by viridans streptococci. Am J Respir Crit Care Med 1997; 156: $1508-1514$

18 Porta G, Rodriguez-Carballeira M, Gomez L, Salavert M, Freixas N, Xercavins M, Garau J. Thoracic infection caused by Streptococcus milleri. Eur Respir J 1998; 12: 357 - 362

${ }^{19}$ Smith CJ, Billingslea TH. The treatment of lung abscesses with penicillin: report of 4 cases. JAMA 1945; 129: 1005-1010

${ }^{20}$ Smith DT. Medical treatment of acute and chronic pulmonary abscesses. J Thorac Surg 1948; 17: 72 - 90

${ }^{21}$ Jensen HE, Amdrup E. Nonspecific abscess of the lung: 129 cases. I. Diagnosis and treatment. Acta Chir Scand 1964; 127: 487-494

${ }^{22}$ Weiss W, Magbojos Q, Flippin HF. Oral penicillin G in the treatment of acute primary nonspecific pulmonary suppuration. Amer J Med Sci 1964; 247: 522 - 530

${ }^{23}$ Abernathy RS. Antibiotic therapy of lung abscesses: effectiveness of penicillin. Dis Chest 1968; 53: $592-598$

${ }^{24}$ Weiss W, Cherniack NS. Acute nonspecific lung abscess: a controlled study comparing oral and parenterally administered penicillin G. Chest 1974; 66: 348 - 351

25 Bartlett JG, Gorbach SL. Treatment of aspiration pneumonia and primary lung abscess, penicillin G vs clindamycin. JAMA 1975; 234: 935 - 937

${ }^{26}$ Levison ME, Mangura CT, Lorber B, Abrutyn E, Pesanti EL, Levy RS, Macgregor R, Schwartz AR. Clindamycin compard with penicillin for the treatment of anaerobic lung abscess. Ann Intern Med 1983; 98: 466 - 471

${ }^{27}$ Gudiol F, Manresa F, Pallares R, Dorca J, Rufi G, Boada J, Ariza X, Casanova A, Villadrich PF. Clindamycin vs penicillin for anaerobic lung infections. Arch Intern Med 1990; 150: 2525 - 2529

28 Perlino CA. Metronidazole versus clindamycin treatment of anaerobic pulmonary infection: failure of metronidazole therapy. Arch Intern Med 1981; 141: 1424-1427

${ }^{29}$ Sanders CV, Hanna BJ, Lewis AC. Metronidazole in the treatment of anaerobic lung infections. Am Rev Respir Dis 1979; 120: 337 343

${ }^{30}$ Germaud P, Poirer J, Jacqueme P, Guerin JCS, Benard Y, Boutin C, Brambilla C, Escailla R, Zuck P. Monotherapy using amoxicillin/ clavulanic acid as a treatment of first choice in communityacquired lung abscess. Rev Pneumol Clin 1993; 49: 137-141

${ }^{31}$ Bartlett JG. Anaerobic bacterial infections of the lung and pleural space. Clin Infect Dis 1993; 16 (Suppl 4): 248-255

${ }^{32}$ Block AJ, Wagley PF, Fisher AM. Delayed closure of lung abscess: a reevaluation of the indication for surgery. John Hopkins Med 1969; 125: $19-24$

${ }^{33}$ Weiss W. Cavity behaviour in acute, primary nonspecific lung abscess. Am Rev Respir Dis 1973; 108: 1273-1275

${ }^{34}$ Hammer DL, Aranda CP, Galati V, Adams FV. Massive intrabronchial aspiration of contents of pulmonary abscess after fiberoptic bronchoscopy. Chest 1978; 74: 306-307

${ }^{35}$ Klein JS, Schultz S, Heffner JE. Interventional radiology of the Chest: image-guided percutaneous drainage of pleural effusions, lung abscess, and pneumothorax. AJR 1995; 164: 581 - 588

${ }^{36}$ Metras H, Chapin J. Lung abscess and bronchial catheterization. J Thorac Surg 1954; 27: 157 - 159

${ }^{37}$ Connors JP, Roper CL, Ferguson TB. Transbronchial catheterization of pulmonary abscesses. Ann Thorac Surg 1975; 19: $254-$ 260

${ }^{38}$ Rowe LD, Keane WM, Jafek BW, Atkins Jr JP. Transbronchial drainage of pulmonary abscesses with the flexible fiberoptic bronchoscope. Laryngoscope 1979; 89: 122-128

39 Jeong MP, Kim WS, Han SK, Shim YS, Kim KY, Han YC. Transbronchial catheter drainage via fiberoptic bronchoscopy in tractable lung abscess. Korean J Intern Med 1989; 4: 54-58
${ }^{40}$ Schmitt GS, Ohar JM, Kanter KR, Naunheim KS. Indwelling transbronchial catheter drainage of pulmonary abscess. Ann Thorac Surg 1988; 45: 43-47

${ }^{41}$ Vainrub B, Musher DM, Guinn GA, Young EJ, Septimus EJ, Travis LL. Percutaneous drainage of lung abscess. Am Rev Respir Dis 1978; 117: $153-160$

42 Yellin A, Yellin EO, Lieberman Y. Percutaneous tube drainage: the treatment of choice for refractory lung abscess. Ann Thorac Surg 1985; 39: 267-270

${ }^{43}$ Rice TW, Ginsberg RJ, Todd TR. Tube drainage of lung abscesses. Ann Thorac Surg 1987; 44: 356 - 359

${ }^{44}$ Parker LA, Melton JW, Delany DJ, Yankaskas BC. Percutaneous small bore catheter drainage in the managment of lung abscesses. Chest 1987; 92: $213-218$

${ }^{45}$ Shim C, Santos GH, Zelefsky M. Percutaneous drainage of lung abscess. Lung 1990; 168: $201-207$

${ }^{46}$ van Sonnenberg E, D'Agostino HB, Casola G, Wittich GR, Varney C. Lung abscess: CT-guided drainage. Radiology 1991; 178: $347-$ 351

${ }^{47}$ Ha HK, Kang MW, Park JM, Shinn KS, Bahk YW. Lung abscess. Percutaneous catheter therapy. Acta Radiol 1993; 34: 362 - 365

${ }^{48}$ Monaldi V. Endocavitary aspiration in the treatment of lung abscess. Dis Chest 1965; 29: $193-201$

${ }^{49}$ Cameron EWJ, Whitton ID. Percutaneous drainage in the treatment of Klebsiella pneumoniae lung abscess. Thorax 1977; 32: $673-676$

${ }^{50}$ Weinberg D. Percutaneous drainage of lung abscess. J Thorac Cardiovasc Surg 1984; 87: 308-312

${ }^{51}$ Potgieter PD, Hamond JM, Musson G, Odell J. Surgical drainage of lung abscess complicating acute community-acquired pneumonia. Chest 1991; 99: 1280-1282

${ }^{52}$ Baker CE, Herbend LW, Oddson TA, Putnam CE. Differentiation of empyemas from periperal pulmonary abscesses: the value of computed tomography. Radiology 1980; 135: $755-758$

${ }^{53}$ Thoms NW, Puro HE, Arbulu A. The significance of hemoptysis in lung abscess. J Thor Cardiovasc Surg 1970; 59: 617-629

${ }^{54}$ Thoms NW, Wilson RF, Puro HE, Arbulu A. Life-threatening hemoptysis in primary lung abscess. Ann Thorac Surg 1972; 14: $347-358$

Priv.-Doz. Dr. med. S. Ewig

Medizinische Universitäts-Poliklinik

Wilhelmstraße 35

53111 Bonn

E-mail: santiago.ewig@ukb.uni-bonn.de 